\title{
Broadband emission from a multicore fiber fabricated with granulated oxides
}

\author{
Loredana Di Labio, ${ }^{1, *}$ Willy Lüthy, ${ }^{1}$ Valerio Romano, ${ }^{1}$ Frédéric Sandoz, ${ }^{2}$ \\ and Thomas Feurer ${ }^{1}$ \\ ${ }^{1}$ Institute of Applied Physics, University of Bern, Sidlerstrasse 5, CH-3012 Bern, Switzerland \\ ${ }^{2}$ Silitec Fibers SA, Route de la Gare 70, CH-2017 Boudry, Switzerland \\ *Corresponding author: loredana.dilabio@iap.unibe.ch
}

Received 5 December 2007; accepted 1 February 2008;

posted 13 February 2008 (Doc. ID 90555); published 31 March 2008

\begin{abstract}
We demonstrate a multicore multidopant fiber which, when pumped with a single pump source around $\sim 800 \mathrm{~nm}$, emits a more than one octave-spanning fluorescence spectrum ranging from 925 to $2300 \mathrm{~nm}$. The fiber preform is manufactured from granulated oxides and the individual cores are doped with five different rare earths, i.e., $\mathrm{Nd}^{3+}, \mathrm{Yb}^{3+}, \mathrm{Er}^{3+}, \mathrm{Ho}^{3+}$, and $\mathrm{Tm}^{3+}$. C 2008 Optical Society of America OCIS codes: $\quad 060.2280,060.2290,060.2400,160.5690,300.2530,300.6340$.
\end{abstract}

\section{Introduction}

Broadband light sources are a prerequisite for a large variety of applications, such as spectroscopy [1], microscopy [2], sensing [3], or medical diagnosis to name a few. Ōne prominent example is optical coherence tomography [4]. Many of the applications rely on the extremely short coherence length, which is a consequence of the broad spectral distribution and which may be as short as a few micrometers. Commonly employed broadband light sources are thermal light sources, light emitting diodes, superluminescence diodes [5], amplified spontaneous emission and superfluorescence fiber sources [6], femtosecond oscillators, or white light sources based on a nonlinear continuum generation [7]. Other sources, such as very long Raman fiber lasers [8], have been investigated but are not as widespread. While most light sources have bandwidths of $<100 \mathrm{~nm}$, some are as broad as a couple of hundred nanometers. Because of their superior beam quality and high spatial coherence fiber based sources, most prominently superfluorescent rare earth-doped or highly nonlinear fibers, are often preferred to other sources. A further important characteristic is the

0003-6935/08/101581-04\$15.00/0

(C) 2008 Optical Society of America output power of a light source. Generally, the broadest bandwidth but also the lowest output power is reached with spontaneous emission. Amplified spontaneous emission has a higher power but shows some narrowing of the spectra depending on the degree of amplification. Finally, the highest output power is reached with laser emission, but in continuous wave (cw) operation this comes at the cost of a bandwidth that is considerably reduced. Nevertheless, even in the case of cw laser activity, laser emission can cover a range of $50 \mathrm{~nm}$ in the case of an $\mathrm{Nd}^{3+}: \mathrm{Al}^{3+}$ : glass fiber $[9,10]$ or $75 \mathrm{~nm}$ for a $\mathrm{Yb}^{3+}: \mathrm{Al}^{3+}$ : glass fiber [11]. The broadest bandwidths and the highest output powers, however, are undoubtedly reached with standalone mode-locked oscillators or with subsequent continuum generation at the expense of high costs. Here, we report on an alternative approach to realize an extremely broadband cw fluorescence light source, which is based on a fiber with multiple-doped cores pumped by a single pump source. Multicore fibers have been previously reported with an emphasis on enlarging the effective mode area for high power laser applications [12-14]; the cores were either doped with $\mathrm{Nd}^{3+}$ or $\mathrm{Yb}^{3+}$ and the fibers were not intended for broadband light generation. The reported approach has the advantage that the generated emission can be directly guided to the 
application. Further the small emitting area and the limited numerical aperture allow for reaching a high brilliance. For practical reasons the experiments were performed with a Ti:sapphire laser but the arrangement could also be operated with the use of a single-stripe diode laser [15]. Therefore it has the potential of a very compact tool for applications requiring a broadband light source.

\section{Fabrication Procedure}

The geometry of our design is shown in Fig. 1(a). Seven differently doped cores are arranged in a honeycomb geometry with six cores surrounding the central core. With the goal of covering an emission band ranging from $\sim 900 \mathrm{~nm}$ to over $2 \mu \mathrm{m}$, five different trivalent rare earth ions have been chosen, i.e., $\mathrm{Nd}^{3+}, \mathrm{Ho}^{3+}, \mathrm{Er}^{3+}, \mathrm{Tm}^{3+}$, and $\mathrm{Yb}^{3+}$. They are well suited because all can be excited with a single pump wavelength of $\sim 800 \mathrm{~nm}$ [16]. Only $\mathrm{Yb}^{3+}$ has its maximum absorption at $97 \overline{\mathrm{n} m}$ but the transition is so broad that even at $800 \mathrm{~nm}$ the absorption is sufficiently high. The emission spectra cover the range from $925 \mathrm{~nm}\left(\mathrm{Nd}^{3+4} F_{5 / 2} \rightarrow{ }^{4} I_{11 / 2}\right)$ to $2100 \mathrm{~nm}$ $\left(\mathrm{Ho}^{3+5} I_{7} \rightarrow{ }^{5} I_{8}\right)$. The doping concentration is chosen to yield an absorption length of $\sim 20 \mathrm{~cm}$ at the pump wavelength of $800 \mathrm{~nm}$. With the $\mathrm{Nd}^{3+}, \mathrm{Er}^{3+}$, and $\mathrm{Tm}^{3+}$ absorption cross sections from $[17,18]$ concentrations of $0.018,0.342$, and 0.235 at. $\%$ are used. For $\mathrm{Ho}^{3+}$ and $\mathrm{Yb}^{3+}$ a concentration of 1 at. $\%$ is chosen. To facilitate easy identification of the differently doped cores, two are doped with $\mathrm{Nd}^{3+}$.

The fibers are drawn from preforms that are fabricated with granulated oxides $[9,10,19]$. In a first step the preform is assembled by closely stacking seven silica tubes of $3 \mathrm{~mm} \times 5 \mathrm{~mm}$ diameter in the center of a bigger $17 \mathrm{~mm} \times 21 \mathrm{~mm}$ silica tube and by filling the remaining space with undoped granulated silica of a typically $400 \mu \mathrm{m}$ grain size as described in detail in $[9,10]$. Each inner tube is filled with a mixture of granulated silica, the appropriate concentration of rare earth oxide, and aluminum oxide. Aluminum prevents the rare earth ions from clustering and raises the index of refraction to facilitate the guiding of light. The aluminum oxide

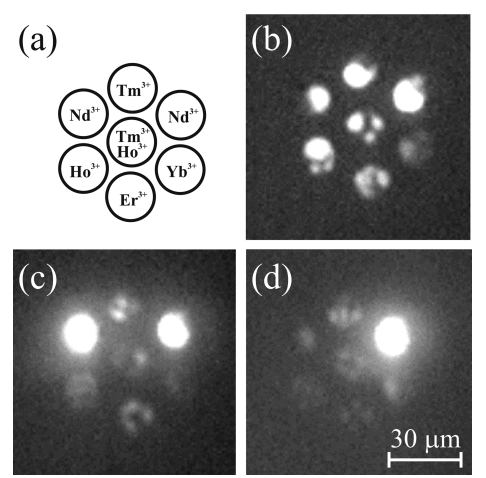

Fig. 1. (a) Arrangement of the seven differently doped cores. Images of the rear fiber end with (b) all cores pumped, (c) two $\mathrm{Nd}^{3+}$-doped cores pumped, and (d) single $\mathrm{Nd}^{3+}$-doped core pumped. concentration corresponds to 7 at. $\%$ of $\mathrm{Al}^{3+}$ with respect to silicon. The preform is preheated at $\sim 1400^{\circ} \mathrm{C}$, evacuated for $2 \mathrm{~h}$, and then drawn at a temperature of $\sim 1850^{\circ} \mathrm{C}$ to a fiber with a diameter of $1.24 \mathrm{~mm}$. In a second step, this fiber is then packed in the center of a larger silica tube $(17 \mathrm{~mm} \times 21 \mathrm{~mm})$ and the remaining space is again filled with undoped granulated silica. After preheating and evacuation the final preform is drawn to a fiber with diameters ranging from 1.45 to $0.51 \mathrm{~mm}$ corresponding to core diameters of 16 to $5 \mu \mathrm{m}$, respectively. Thus, all cores are multimode, except for the smallest diameter of $5 \mu \mathrm{m}$, where the limit for a single-mode operation for all wavelengths of interest is reached.

\section{Experimental}

In the first experiment a $21 \mathrm{~cm}$ long fiber with $16 \mu \mathrm{m}$ large cores is selected and the seven cores are pumped either simultaneously or individually. The pump light of $\sim 800 \mathrm{~nm}$ stems from an argon-ion laser pumped Ti:sapphire system (Spectra Physics, Model 3900 ) with a maximum pump power of $400 \mathrm{~mW}$. The pump laser is focused with a lens $(f=14.5 \mathrm{~mm})$ to a spot size of $\sim 30 \mu \mathrm{m}$ onto the front face of the fiber and the fluorescence is detected at the rear end. Prior to all the spectral measurements, the rear end of the fiber is imaged onto a CCD camera, and the pump light is aligned until either all or a selection of cores is pumped. Since the camera is equipped with a silicon-based CCD and protected with a $6 \mathrm{~mm}$ RG 1000 Schott filter its sensitivity is restricted to wavelengths between 900 and $1150 \mathrm{~nm}$. Nevertheless, emission from all rare earth ions may be detected because they all have transitions within this range. Figure 1(b) shows the rear end of the fiber with all cores simultaneously excited. The emission from the different cores is assigned to $\mathrm{Yb}^{3+}\left({ }^{2} F_{5 / 2} \rightarrow\right.$ $\left.{ }^{2} F_{7 / 2}\right), \quad \mathrm{Nd}^{3+}\left({ }^{4} F_{3 / 2} \rightarrow{ }^{4} I_{11 / 2}\right), \operatorname{Er}^{3+}\left({ }^{4} I_{11 / 2} \rightarrow{ }^{4} I_{15 / 2}\right)$, $\mathrm{Ho}^{3+}\left({ }^{5} I_{5} \rightarrow{ }^{5} I_{8}\right.$ or $\left.{ }^{5} I_{6} \rightarrow{ }^{5} I_{8}\right)$, and $\operatorname{Tm}^{3+}\left({ }^{3} H_{5} \rightarrow\right.$ ${ }^{3} \mathrm{H}_{6}$ or ${ }^{3} \mathrm{H}_{4} \rightarrow{ }^{3} \mathrm{H}_{6}$ ), respectively. In Fig. 1(c) only the two $\mathrm{Nd}^{3+}$-doped cores are pumped by aiming the pump light at the upper half of the core area. Finally, Fig. 1(d) demonstrates the controlled excitation of only a single core $\left(\mathrm{Nd}^{3+}\right)$ by focusing and aiming the pump light properly. To record the spectrum of a selectively pumped fiber core, its output is collimated with a $20 \times$ microscope objective and imaged with a second lens $(f=14.5 \mathrm{~mm})$ onto the aperture of a fiber-coupled spectrometer. Depending on the wavelength range three different spectrometers (AVS-USB2000 for 650-1100 nm, AvaSpec-NIR2561.7 for $900-1700 \mathrm{~nm}$, and Ocean Optics NIR256-2.5 for $900-2500 \mathrm{~nm}$ ) are used. The pump light is suppressed with a $4 \mathrm{~mm}$ RG 1000 Schott filter. The measured spectra of individually as well as simultaneously pumped cores are shown in Fig. 2 . When only the $\mathrm{Yb}^{3+}$-doped core is pumped [see Fig. 2(a)], the well-known fluorescence spectrum of $\mathrm{Yb}^{3+}$ is observed with the prominent sharp feature of the ${ }^{2} F_{5 / 2} \rightarrow{ }^{2} F_{7 / 2}$ transition at $977 \mathrm{~nm}$. The spectrum 

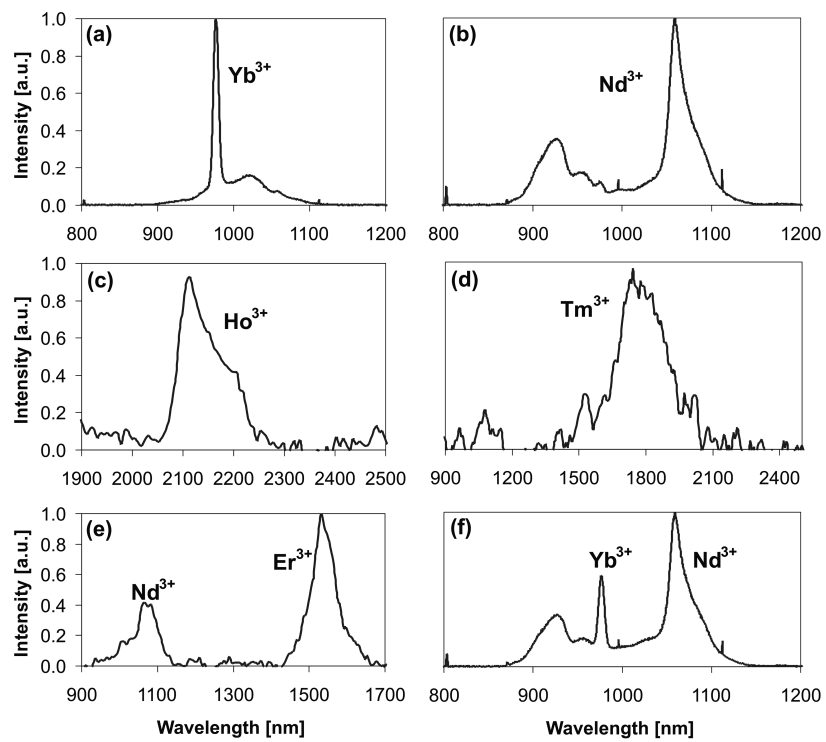

Fig. 2. Spectra of individually pumped $16 \mu \mathrm{m}$ large cores: (a) $\mathrm{Yb}^{3+}$, (b) $\mathrm{Nd}^{3+}$, (c) $\mathrm{Ho}^{3+}$, and (d) $\mathrm{Tm}^{3+}$. When pairs of cores are simultaneously pumped mixed spectra are observed: (e) $\mathrm{Nd}^{3+}$ and $\mathrm{Er}^{3+}$ and (f) $\mathrm{Nd}^{3+}$ and $\mathrm{Yb}^{3+}$.

of the $\mathrm{Nd}^{3+}$-doped core [see Fig. 2(b)] shows the ${ }^{4} F_{3 / 2} \rightarrow{ }^{4} I_{11 / 2}$ transition at $1060 \mathrm{~nm}$, and the fluorescence of $\sim 930 \mathrm{~nm}$ stems from the ${ }^{4} F_{3 / 2} \rightarrow{ }^{4} I_{9 / 2}$ transition. The emission spectra of the $\mathrm{Ho}^{3+}$-doped core with the ${ }^{5} I_{7} \rightarrow{ }^{5} I_{8}$ transition centered at $2113 \mathrm{~nm}$ and of the $\mathrm{Tm}^{3+}$-doped core with the ${ }^{3} F_{4} \rightarrow$ ${ }^{3} \mathrm{H}_{6}$ transition at $\sim 1.8 \mu \mathrm{m}$ are shown in Figs. $2(\mathrm{c})$ and $2(\mathrm{~d})$. The spectra depicted in Figs. $2(\mathrm{e})$ and $\underline{2(\mathrm{f})}$ are measured with two cores being simultaneously excited. Figure $\underline{2(\mathrm{e})}$ shows the $\mathrm{Er}^{3+}{ }^{4} I_{13 / 2} \rightarrow{ }^{4} I_{15 / 2}$ transition centered at $1532 \mathrm{~nm}$ and the $\mathrm{Nd}^{3+}{ }^{4} F_{3 / 2} \rightarrow$ ${ }^{4} I_{11 / 2}$ transition. The combined excitation of the $\mathrm{Nd}^{3+}$ - and the $\mathrm{Yb}^{3+}$-doped core yields the spectrum in Fig. 2(f). Thus, each excited core separately shows

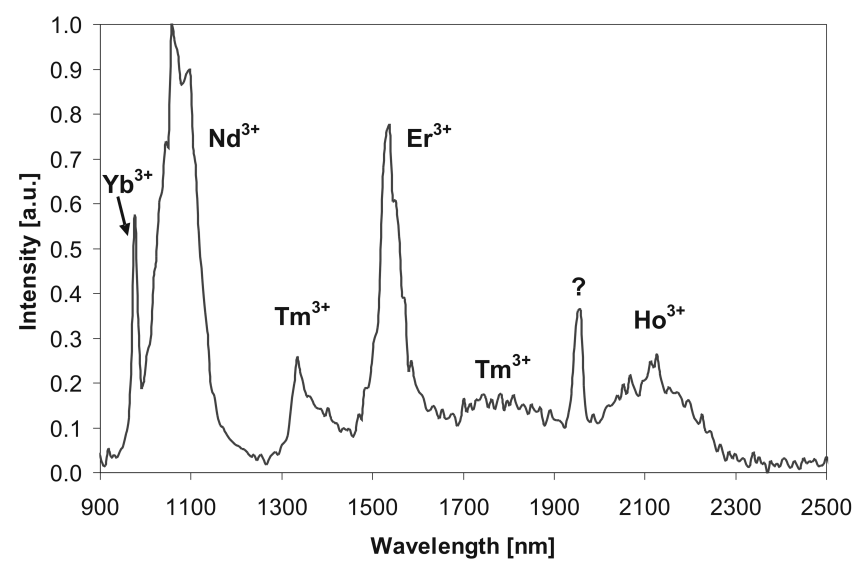

Fig. 3. Fluorescence spectrum emitted from a fiber with $5 \mu \mathrm{m}$ large cores. The spectral intensities are as measured, that is, without correcting for efficiencies. The curve at $1954 \mathrm{~nm}$ labeled with a ? is assigned to a second order peak of the $977 \mathrm{~nm} \mathrm{Yb}^{3+}$ emission. the expected fluorescence proving that the ions are properly ionized and well embedded in the glass matrix. In other words, fabricating cores from granulated oxides seems feasible at least for all the rare earth ions used in this experiment.

In the second experiment a fiber with $5 \mu \mathrm{m}$ large cores is used. The smaller core size allows for homogeneous pumping of all cores without changing the optical setup. Figure 3 shows the spectrum for a pump wavelength of $804 \mathrm{~nm}$. The grating of the spectrometer (Ocean Optics NIR256-2.5) has only 150 lines $/ \mathrm{mm}$ leading to feeble diffraction at the apertures of the grating and consequently to extremely weak and throughout negligible second order contributions. The spectrum indicates contributions from all five rare earths. Note, each core independently emits its spectrum and is largely undisturbed from the others. The width of the resulting spectrum corresponds to one octave plus a major third and nearly completely covers the wavelength range from 925 to $\sim 2300 \mathrm{~nm}$. Only below $1300 \mathrm{~nm}$ there is a gap between the emission of $\mathrm{Nd}^{3+}$ and $\mathrm{Tm}^{3+}$. The spectral gap may be filled with, e.g., $\mathrm{Bi}^{3+}$, which shows fluorescence at $1250 \mathrm{~nm}$ when pumped at $800 \mathrm{~nm}$ [20]. The relative spectral distribution can be considerably modified if the pump wavelength is changed. While a longer wavelength, e.g., $\sim 820 \mathrm{~nm}$, favors the $\mathrm{Yb}^{3+}$ fluorescence and reduces the $\mathrm{Nd}^{3+}$ fluorescence, a shorter wavelength, e.g., $786 \mathrm{~nm}$, strongly favors $\mathrm{Tm}^{3+}$ and restrains the fluorescence from the other ions. That is, the overall spectral distribution can be manipulated within certain limits by a judicious choice of the relative rare earth ion concentrations and the pump wavelength. The efficiency of the broadband light source is mostly limited by the small solid angle that overlaps with the numerical aperture of the fiber, which is roughly between $0.1 \%$ and $1 \%$.

\section{Conclusion}

To conclude, we have demonstrated a seven-core fiber where each core is fabricated from granulated silica mixed with the corresponding rare earth oxide and aluminum oxide. The dopants were selected so that all can be pumped with a single pump laser and simultaneously cover the widest possible fluorescence spectrum. Each core showed the expected fluorescence proving that the ions are properly ionized and embedded in the glass matrix. When all cores are simultaneously excited, the emitted spectrum ranges from 925 to $2300 \mathrm{~nm}$. We would like to emphasize that in the current design different parts of the spectrum originate from different although well-defined spatial areas in the fiber's core region. To avoid that, we are currently working on an alternative design that allows extraction of the whole spectrum from a single core.

We thank D. Weber for technical assistance and M. Mühlheim, M. Neff, and R. Scheidegger for their support with the fiber drawing. This work was supported in part by the Swiss Commission for the Encouragement of Scientific Research CTI under 
project 7864.1 .

\section{References}

1. M. Jacquemet, N. Picqué, G. Guelachvili, A. Garnache, I. Sagnes, M. Strassner, and C. Symonds, "Continuous-wave $1.55 \mu \mathrm{m}$ diode-pumped surface emitting semiconductor laser for broadband multiplex spectroscopy," Opt. Lett. 32, 13871389 (2007).

2. T. R. Corle and G. S. Kino, Confocal Scanning Optical Microscopy and Related Imaging Systems (Academic, 1996).

3. S. Martin-Lopez, M. Gonzalez-Herraez, A. Carrasco-Sanz, F. Vanholsbeeck, S. Coen, H. Fernandez, J. Solis, P. Corredera, and M. L. Hernanz, "Broadband spectrally flat and high power density light source for fibre sensing purposes," Meas. Sci. Technol. 17, 1014-1019 (2006).

4. A. F. Fercher, W. Drexler, C. K. Hitzenberger, and T. Lasser, "Optical coherence tomography-principles and applications," Rep. Prog. Phys. 66, 239-303 (2003).

5. V. R. Shidlovski and J. Wei, "Superluminescent diodes for optical coherence tomography," Proc. SPIE 4648, 139147 (2002).

6. F. Gan and Y. Chen, "The spectral characteristics of superfluorescence in rare-earth-doped silica fibres," Pure Appl. Opt. 2, 359-365 (1993).

7. G. Genty, S. Coen, and J. M. Dudley, "Fiber supercontinuum (Invited)," J. Opt. Soc. Am. B 24, 1771-1785 (2007).

8. W. Zhang, Y. Wang, J. Peng, and X. Liu, "Broadband high power continuous wave fiber Raman source and its applications," Opt. Commun. 231, 371-374 (2004).

9. R. Renner-Erny, L. Di Labio, and W. Lüthy, "A novel technique for active fibre production," Opt. Mater. 29, 919-922 (2007).

10. L. Di Labio, R. Renner-Erny, P. Blattnig, V. Romano, W. Lüthy, F. Sandoz, and T. Feurer, "Novel technology to fabricate mixed multi-core fibre lasers in standard and air-clad configuration," presented at the Second European Physical Society, Quantum Electronics and Optics Division, Europhoton Conference, Pisa, Italy, 10-15 September 2006.

11. U. Pedrazza, V. Romano, and W. Lüthy, "Yb3 ${ }^{3+}: \mathrm{Al}^{3+}$ : sol-gel silica glass fibre laser," Opt. Mater. 29, 905-907 (2007).

12. P. Glas, M. Naumann, A. Schirrmacher, and T. Pertsch, "The multicore fiber-a novel design for a diode pumped fiber laser," Opt. Commun. 151, 187-195 (1998).

13. P. K. Cheo, A. Liu, and G. G. King, "A high-brightness laser beam from a phase-locked multicore Yb-doped fiber laser array," IEEE Photon. Technol. Lett. 13, 439-441 (2001).

14. Y. Huo and P. K. Cheo, "Analysis of transverse mode competition and selection in multicore fiber lasers," J. Opt. Soc. Am. B 22, 2345-2349 (2005).

15. W. Lüthy and H. P. Weber, "High-power monomode fiber lasers," Opt. Eng. 34, 2361-2364 (1995).

16. A. A. Kaminskii, Laser Crystals (Springer Verlag, 1981), p. 10.

17. P. Tosin, W. Lüthy, and H. P. Weber, "Determination of the spectral absorption in silica samples with known rare earth dopant concentration," in Proceedings of the Ninth International Conference on Modern Materials and Technologies, World Ceramics Congress and Forum of New Materials, Florenz, Italy, 14-19 June 1998.

18. S. Zemon, B. Pedersen, G. Lambert, W. J. Miniscalco, L. J. Andrews, R. W. Davies, and T. Wei, "Excited-state absorption cross-sections in the $800 \mathrm{~nm}$ band for Er-doped, Al/P-silica fibres: measurements and amplifier modeling," IEEE Photon. Technol. Lett. 3, 621-624 (1991).

19. J. Ballato and E. Snitzer, "Fabrication of fibres with high rareearth concentrations for Faraday isolator applications," Appl. Opt. 34, 6848-6854 (1995).

20. T. Suzuki and Y. Ohishi, "Ultrabroadband near-infrared emission from Bi-doped $\mathrm{Li}_{2} \mathrm{O}-\mathrm{Al}_{2} \mathrm{O}_{3}-\mathrm{SiO}_{2}$ glass," Appl. Phys. Lett. 88, 191912-1-191912-3 (2006) 